\title{
靛红并范醌二甲酰亚胺类共轭分子的设计合成及性质研究
}

\author{
谭 丹 吴 赛 $^{a}$ 魏 欢 ${ }^{b}$ 胡袁源 ${ }^{b}$ 陈华杰*,a \\ ( ${ }^{a}$ 湘潭大学化学学院 环境友好化学与应用教育部重点实验室 湖南湘潭 411105) \\ ( ${ }^{b}$ 湖南大学物理与微电子科学学院 微纳光电器件及应用教育部重点实验室 长沙 410082)
}

\begin{abstract}
摘要 将靛红和氮杂靛红衍生物单元分别与苊醌二甲酰亚胺进行 Knoevenagel 缩合, 开发了两个结构新型、强缺电特 性的异靛青衍生物受体单元，包括靛红并范醌二甲酰亚胺单元(A1)和氮杂靛红并范醌二甲酰亚胺单元(A2). 然后运用 “双受体策略”，进一步设计并合成了两个含 $\mathbf{A 1}$ 和 A2 受体单元的 A-A 型二联体共轭分子(BA1 和 BA2), 并对比研究 了吡啶氮原子对共轭分子的骨架结构、吸收光谱、能级结构以及载流子迁移率的影响. 研究发现: 相比经典的异靛蓝 受体, $\mathbf{A 1}$ 和 $\mathbf{A 2}$ 受体单元拥有更低的 LUMO 能级 $(c a .-4.0 \mathrm{eV})$ 和更大的骨架共轭; 相比 $\mathbf{A 1}$ 和 $\mathbf{A 2}$, 二联体化合物 BA1 和 BA2 不仅拥有更大的骨架共轭和更好的分子对称性，而且在整个紫外-可见光区的展现出强的光捕获能力; 吡啶氮 原子的引入增强了 $\mathbf{A 2}$ 和 $\mathbf{B A 2}$ 的骨架共平面性以及电子亲和力, 有效地降低了 $\mathbf{A 2}$ 和 BA2 的 HOMO 和 LUMO 能级值. 基于 BA1 和 BA2 薄膜, 制备了 $n$-型场效应晶体管器件, 其电子迁移率分别达 $1.64 \times 10^{-3}$ 和 $2.52 \times 10^{-3} \mathrm{~cm}^{2} \cdot \mathrm{V}^{-1} \cdot \mathrm{s}^{-1}$. 关键词 靛红; 范醌二甲酰亚胺; 异靛青; 电子迁移率
\end{abstract}

\section{Design, Synthesis, and Properties of Conjuated Molecules with Isatin-Fused Acenaphthenequinone Imide Moieties}

\author{
Tan, Dan $^{a} \quad \mathrm{Wu}, \mathrm{Sai}^{a} \quad$ Wei, Huan ${ }^{b} \quad \mathrm{Hu}$, Yuanyuan $^{b} \quad$ Chen, Huajie ${ }^{*, a}$ \\ $\left({ }^{a}\right.$ Key Laboratory of Environmentally Friendly Chemistry and Application of Ministry of Education, College of Chemistry, \\ Xiangtan University, Xiangtan, Hunan 411105) \\ $\left({ }^{b}\right.$ Key Laboratory for Micro/Nano Optoelectronic Devices of Ministry of Education, School of Physics and Electronics, \\ Hunan University, Changsha 410082)
}

\begin{abstract}
Two highly electron-deficient, novel isoindigo (IID) derivative acceptor units, including isatin-fused acenaphthenequinone imide (A1) and nitrogen-doped isatin-fused acenaphthenequinone imide (A2), were designed and synthesized via Knoevenagel consendation reaction. In comparison with the well-known IID unit, both A1 and A2 acceptor units exhibit reduced LUMO energy levels $(c a .-4.0 \mathrm{eV})$ and extended $\pi$-conjugation backbone owing to the incorporation of strongly electron-withdrawing acenaphthenequinone imide. The properties observed here for both $\mathbf{A} 1$ and $\mathbf{A 2}$ are thus indicative of promising potential in the development of $n$-type organic semiconductors. On the basis of both $\mathbf{A 1}$ and $\mathbf{A 2}$ acceptor units, two A-A type organic $\pi$-conjugated molecules (BA1 and BA2) were further designed and synthesized by self-coupling of two identical $\mathbf{A 1}$ or $\mathbf{A 2}$ acceptor units. The effect of pyridal nitrogen on the backbone structure, optical absorption, energy level, and carrier mobility of the as-prepared $\pi$-conjugated molecules is studied systematically. The comparative investigation reveals that self-coupling of dual acceptor units into BA1 and BA2 not only endows them with extended conjugation backbone and enhanced molecular symmetry, but also improves their light-capturing abilities in the whole ultraviolet-visible region as relative to their parent acceptor units (A1 and A2). Moreover, the pyridal N-containing BA1 and BA2 possess enhanced backbone coplanarity and electron affinity as compared to their parent units (A1 and A2), thereby leading to reduced HOMO and LUMO energy levels. Finally, $n$-type thin-film transistors are further fabricated by adopting both BA1 and BA2 as the active layers, affording the electron motilities of $1.64 \times 10^{-3}$ and $2.52 \times 10^{-3} \mathrm{~cm}^{2} \cdot \mathrm{V}^{-1} \cdot \mathrm{s}^{-1}$, respectively.

Keywords isatin; acenaphthenequinone imide; isoindigo; electron mobility
\end{abstract}

靛红是一类重要的化学原料, 被广泛用于制备药物 中间体 ${ }^{[1]}$ 、有机染料 ${ }^{[2-3]}$ 以及有机共轭材料 ${ }^{[4-7]}$. 化学家将

* Corresponding authors. E-mail: chenhjoe@163.com; chenhjoe@xtu.edu.cn

Received May 14, 2020; revised June 12, 2020; published online June 24, 2020.

Project supported by the National Natural Science Foundation of China (No. 21875202), the Hunan Provincial Natural Science Foundation of China (No. 2018JJ1024), and the Science and Technology Planning Project of Hunan Province (No. 2017RS3048).

国家自然科学基金(No. 21875202)、湖南省自然科学基金杰出青年基金(No. 2018JJ1024)和湖南省科技厅计划(No. 2017RS3048)资助项目. 
靛红分子中的酮羰基 $(\mathrm{C}=\mathrm{O})$ 两两缩合, 开发了含两个内 酰亚胺单元的异靛青染料 ${ }^{[8-9]}$ (简称 IID, 图 1). 研究发 现, IID 分子具有光热稳定性好、光捕获能力强、电子亲 和力高、共平面性好、溶解性可调以及骨架易于修饰等 优点 ${ }^{[4-6]}$, 因此 IID 及其衍生物被广泛地应用于高性能的 有机场效应晶体管 ${ }^{[4-6]}$ 、有机太阳能电池 ${ }^{[6,10]}$ 、超级电容 器 ${ }^{[7]}$ 和近红外二区光热转换材料 ${ }^{[11]}$ 等领域. 近年来, 对 于 IID 母核的修饰，化学家主要集中在如下三方面：(1) 对 IID 母核外侧苯环进行替换 ${ }^{[12-13]}$ 、杂原子修饰 ${ }^{[14-17]}$ 或 者共轭拓展 ${ }^{[18-20]}$. 譬如, 将 IID 母核外侧苯环用吡啶替 换, 可开发出电子亲和力更高的氮杂 IID 衍生物受体单 元(NIID ${ }^{[14]}$, 图 1); (2)对 IID 母核的中心 $\pi$ 桥进行共轭拓 展 $^{[21-25]}$. 譬如, 将苯并二呋喃二酮引入到 IID 母核的中 心 $\pi$ 桥中, 可开发出骨架共轭更大、缺电子能力更强的 异靛衍生物受体单元(BDOPV) ${ }^{[22]}$; (3)对 IID 母核的溶解 侧链进行调整与修饰 ${ }^{[26-28]}$. 譬如, 改变 IID 母核上溶解 侧链的分叉点, 可有效调控 IID 基聚合物半导体材料的 溶解性、结晶性以及载流子迁移率等性能参数 ${ }^{[27-28]}$. 基 于以上策略, 化学家近年来开发了系列含 IID 及其衍生 物受体的 D-A 型聚合物半导体材料, 并实现了高的空穴 和电子迁移率以及高的光伏效率 ${ }^{[5-6,29]}$.

苍醌二甲酰亚胺(ANQNI，图 1)是另一类经典的缺 电子构建单元 ${ }^{[30-31]}$, 它具有与萘四甲酰二亚胺(NDI) 类 似的化学结构和 LUMO 能级值 $(-3.39 \mathrm{eV}$, 图 1). 近年 来, ANQNI 衍生物已被成功地用于构建有机/聚合物电 子传输材料 ${ }^{[32-33]}$ 、近红外吸收共轭聚合物 ${ }^{[31-32]}$ 以及 A-D-A 型非富勒烯小分子体料 ${ }^{[34-36]}$. 譬如, 化学家将 ANQNI 的两个碳基 $(\mathrm{C}=\mathrm{O})$ 与芳香四胺缩合, 制备出了 系列含吡嗪杂环的芳香酰亚胺类有机/聚合物半导体材

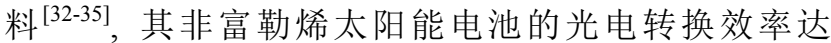
8.0\%以上 ${ }^{[34-35]}$. 我们近期发现, 将 ANQNI 与 1,3-二噻 吩基-2-丙酮进行缩合，可开发出具有近红外光捕获能 力的有机共轭小分子及其 D-A 型共轭聚合物 ${ }^{[31]}$, 其光 学带隙可达 $1.0 \mathrm{eV}$ 以下. 鉴于 ANQNI 分子独特的化学 反应活性和高的电子亲和力, 我们预期将 ANQNI 与靛 红衍生物缩合, 有望开发出骨架共轭大、电子亲和力强 以及光捕获范围宽的 $n$-型有机小分子半导体材料.

基于以上分析，本工作采用 Knoevenagel 反应，将 强缺电特性的 ANQNI 单元与靛红衍生物缩合, 设计并 合成了两类缺电子能力强的新型异靛青衍生物受体单 元, 包括靛红并范醌二甲酰亚胺(A1)和氮杂靛红并范醌 二甲酰亚胺(A2), 通过密度泛函理论(DFT)理论计算发 现: $\mathrm{A1}$ 和 $\mathrm{A2}$ 的计算 LUMO 能级值分别达 -3.14 和 $-3.28 \mathrm{eV}$ ，显著低于经典的异靛青(IID, $-2.66 \mathrm{eV}$ )和 氮杂异靛 $($ NIID, $-2.96 \mathrm{eV})$ 的 LUMO 能级值, 表明 $\mathbf{A 1}$ 和 $\mathbf{A 2}$ 是一类吸电子能力强的 $n$-型有机半导体材料构建 模块. 因此，本工作运用 “A-A 双受体策略”，设计并合 成了两个含 $\mathrm{A} 1$ 和 $\mathrm{A2}$ 受体单元的二联体化合物(BA1 和 BA2), 并对其分子结构、吸收光谱、能级结构以及载流 子传输性能进行对比研究. 将两个不对称的受体单元两 两相连, 希望有效地提高目标分子(BA1 和 BA2)的骨架 共轭、分子对称性以及电子亲和力，同时拓宽目标分子 的光谱吸收范围.

\section{1 结果与讨论}

\section{1 合成与表征}

图 2 给出了新型电子受体单元(A1 和 $\mathbf{A 2})$ 及其二联

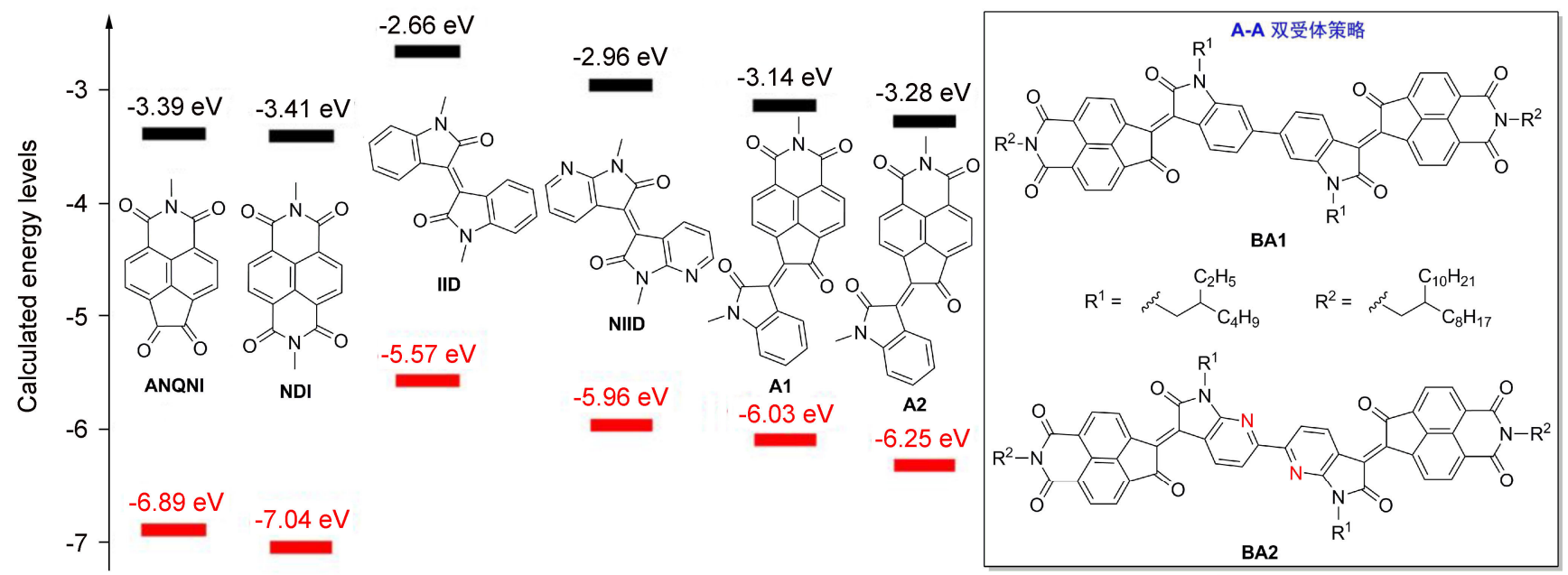

图 1 新设计的靛红并苍醌二甲酰亚胺受体单元(A1 和 A2)和经典受体单元(ANQNI, NDI, IID 和 NIID)间的理论预测的 $\mathrm{HOMO} / \mathrm{LUMO}$ 能级图, 以及含 $\mathbf{A 1}$ 和 $\mathbf{A 2}$ 受体单元的二联体共轭分子(BA1 和 BA2)的结构图

Figure 1 DFT-calculated HOMO/LUMO energy levels for the newly-designed novel isatin-fused acenaphthenequinone imide acceptor (A1 and A2) and four well-known acceptor moieties (ANQNI, NDI, IID, and NIID), and the chemical structures of BA1 and BA2 
体化合物 (BA1 和 BA2)的合成路线. 化合物 C1、C2、 $\mathbf{C 5}$ 和 $\mathbf{C 6}$ 通过商业购买, $\mathbf{C 3}^{[37]} 、 \mathbf{C} 4^{[38]} 、 \mathbf{C} 7^{[39]} 、 \mathbf{C} 9^{[39]} 、$ $\mathbf{C 1 1}^{[37]} 、 \mathrm{C13}^{[40]}$ 和 $\mathbf{C 1 8}^{[41]}$ 参考文献的方法合成. 我们以 静红(C1)或 6-溴靛红(C2)为起始原料, 采用亲核取代反 应分别制备了烷基取代的中间体 C3 和 C4, 其产率分别 达 75\% 和 78\%. 同时, 以 7-氮杂吲哚(C5)或 6-澳-7-氮杂 吲哚(C6)为起始原料, 通过亲核取代反应分别得到了烷 基取代氮杂吲哚 (C7 和 C8). 然后采用氯铬酸吡啶 (PCC)试剂, 进一步将 $\mathbf{C} 7$ 和 C8 分别转变成 1-乙基- $1 H-$ 吡咯并 [2,3-b]吡啶-2,3-二酮(C9)和 6-溴-1-(2-乙基己 基)-1 $H$-吡咯并 $[2,3-b]$ 吡啶-2,3-二酩(C10), 其产率分别 达 $45 \%$ 和 $48 \%$.

为合成目标电子受体 $\mathrm{A1}$ 和 $\mathrm{A2}$ 单元, 中间体 $(\mathrm{C} 3$ 或 C9)分别与水合肼发生 Wolff-Kishner 反应, 得到了含活 性亚甲基的中间体(C11 和 C12). 在对甲基苯磺酸的催 化下, C11 和 C13 进一步发生 Knoevenagel 缩合反应, 以 50\%的产率合成了目标化合物 A1. 采用相似的缩合机
制, C12 与 $\mathbf{C 1 3}$ 缩合得到了目标化合物 $\mathbf{A 2}$, 其产率高达 $55 \%$.

为合成二联体共轭分子 BA1 和 BA2, 上述所得中 间体(C2 和 C10)分别与六甲基二锡烷之间发生 Stille 偶 联反应, 分别得到了二联体化合物(C14 和 C15), 其产 率分别达 $77 \%$ 和 $85 \%$. 在 $120{ }^{\circ} \mathrm{C}$ 高温条件下, 中间体 (C14 或 C15) 分别与水合胇发生 Wolff-Kishner 反应, 得 到了含活性亚甲基的中间体(C16 或 C17). 在对甲基苯 磺酸的催化下, C16 和 C18 进一步发生 Knoevenagel 缩 合反应，以 $39 \%$ 的产率合成了目标化合物 BA1. 采用相 似的缩合反应, $\mathrm{C} 17$ 与 $\mathrm{C} 18$ 缩合得到了目标化合物 BA2, 其产率高达 $43 \%$.

采用核磁共振氢谱和碳谱表征并证实了所得中间 体以及目标产物(A1、A2、BA1 和 BA2)的化学结构. 在 常温下，化合物 BA1 和 BA2 在二氯甲烷、三氯甲烷以 及氯苯等常见溶剂中具有良好的溶解度. 进一步采用热 重分析仪(TGA)和示差扫描量热分析仪(DSC)研究了

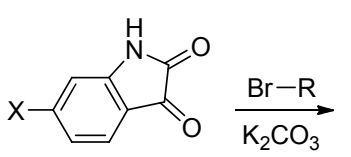

C1, $\mathrm{X}=\mathrm{H}$ C2, $\mathrm{X}=\mathrm{Br}$<smiles>[X]c1ccc2c(c1)N([R])C(=O)C2=O</smiles>

C3, $X=H, R^{1}=$ Ethyl, $75 \%$ C4, $\mathrm{X}=\mathrm{Br}, \mathrm{R}^{1}=$ Ethylhexyl, $78 \%$

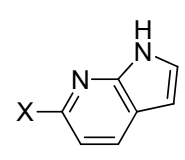

C5, $\mathrm{X}=\mathrm{H}$ C6, $\mathrm{X}=\mathrm{Br}$

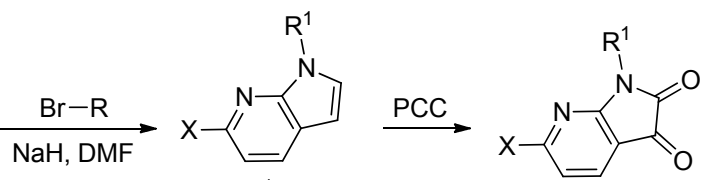

C7, $X=H, R^{1}=$ Ethyl, $85 \%$

C8, $\mathrm{X}=\mathrm{Br}, \mathrm{R}^{1}=$ Ethylhexyl, $88 \%$
C9, $X=H, R^{1}=$ Ethyl, $45 \%$
C10, $X=B r, R^{1}=$ Ethylhexyl,
$48 \%$

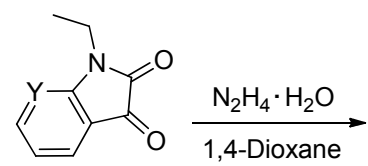

c3, $\mathrm{Y}=\mathrm{CH}$

c9, $Y=N$

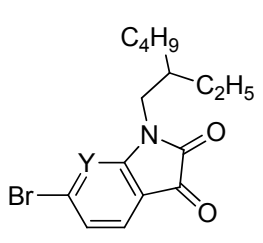

C4, $\mathrm{Y}=\mathrm{CH}$

C10, $Y=N$

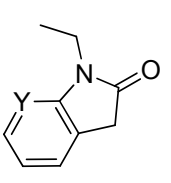

C11, $Y=\mathrm{CH}, 65 \%$

C12, $Y=N, 55 \%$

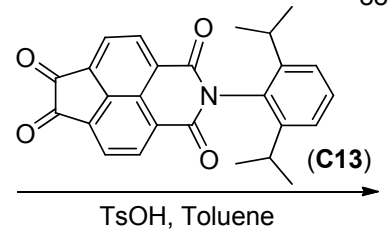

$\mathrm{TsOH}$, Toluene

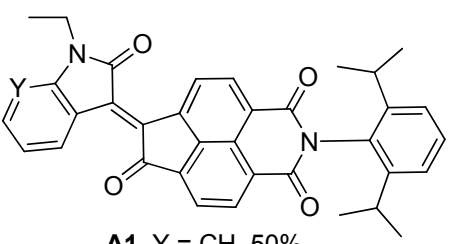

A1, $\mathrm{Y}=\mathrm{CH}, 50 \%$

A2, $Y=N, 55 \%$

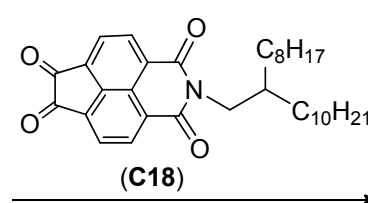

$\mathrm{TsOH}$, toluene

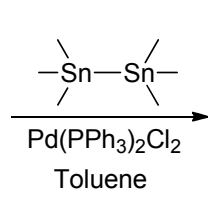

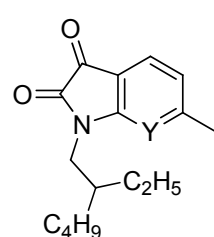
C14, $\mathrm{Y}=\mathrm{CH}, 77 \%$ C15, $Y=N, 85 \%$

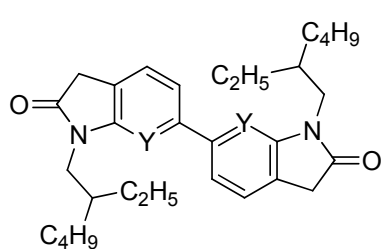

C16, $Y=\mathrm{CH}, 63 \%$ C17, $Y=N, 54 \%$

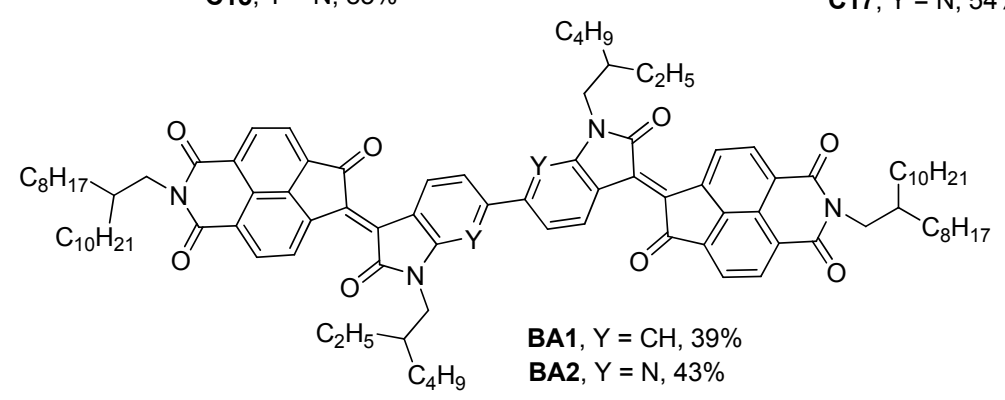

图 2 化合物 $\mathrm{A1}, \mathrm{A2}, \mathrm{BA1}$ 和 $\mathrm{BA2}$ 的合成路线

Figure 2 Synthetic route of the compounds A1, A2, BA1 and BA2 
BA1 和 BA2 的热稳定性和相转变行为. 由 TGA 曲线(见 辅助材料图 S1) 可知: 化合物 BA1 热失重 5\%时对应的 热分解温度 $\left(405{ }^{\circ} \mathrm{C}\right)$ 要明显高于化合物 BA2 $\left(383{ }^{\circ} \mathrm{C}\right)$, 表明化合物 BA1 具有更高的热稳定性. 由 DSC 曲线(见 辅助材料图 S2) 可知: 在升温和降温过程中, 化合物 BA1 和 BA2 均展现出明显的熔融和结晶相转变峰. 其 中, 化合物 BA1 的熔融温度和结晶温度分别处在 233 和 $227{ }^{\circ} \mathrm{C}$ 左右. 相比之下, 化合物 BA2 展现出更高的熔融 温度 $\left(249{ }^{\circ} \mathrm{C}\right)$ 和结晶温度 $\left(246{ }^{\circ} \mathrm{C}\right)$. 这一结果表明: 引 入吡啶氮原子到化合物 BA2 的骨架中, 增加了分子的 刚性和共平面性, 因而获得了更高的熔融/结晶温度.

\section{2 单晶结构分析}

采用溶剂缓慢挥发法, 在室温条件下从甲醇和氯仿 混合溶剂中得到了化合物 $\mathbf{A 2}$ 的单晶, 并采用单晶 $\mathrm{X}$ 射 线衍射技术解析了 $\mathbf{A 2}$ 的晶体结构(图 3), 详细的晶体结 构参数见辅助材料表 S1. 化合物 $\mathbf{A 2}$ 属于三斜晶系, 空 间点阵群为 $P-1$, 晶胞参数分别为 $a=0.90996(3) \mathrm{nm}$, $b=1.73449(6) \mathrm{nm}, c=2.11782(7) \mathrm{nm}$. 从图 3 所示单晶结 构得知: (1)在 $\mathbf{A} 2$ 晶体结构中, 双键 $\pi$ 桥附近的两个羰基 $(\mathrm{C}=\mathrm{O})$ 反向排布, 因而排除了 $\mathrm{A} 2$ 存在骨架异构(反向排 布和同向排布)的可能; (2) $\mathbf{A 2}$ 单晶结构展现出较小的二 面扭转角 $\left(1.38^{\circ}\right)$, 表明 $\mathbf{A} 2$ 分子具有良好的平面性和骨 架共轭结构.

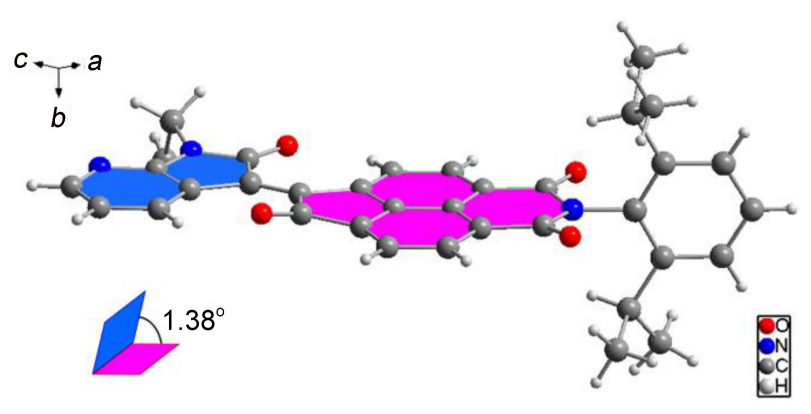

图 3 化合物 $\mathbf{A 2}$ 的单晶结构

Figure 3 Single crystal structure of compound $\mathbf{A 2}$

\section{3 分子结构理论优化}

为探讨分子结构与化合物性质间的关系, 运用 Gaussian 09 在 B3LYP/6-31G(d)机组上优化了所得电子 受体单元(A1 和 A2)及其二联体化合物(BA1 和 BA2)的 分子结构, 并采用密度泛函理论(DFT)计算了化合物的 前线分子轨道能级 ${ }^{[43]}$. 为了简化计算和缩短计算时间, 四个目标化合物中的取代基团用甲基替换. 图 4 给出了 化合物的分子优化构型、电子云分布和前线分子轨道能 级. 对比优化后的分子结构可以得知: A1 骨架中的靛红 与范醌二甲酰亚胺单元之间的扭转二面角达 $10.7^{\circ}$. 在 骨架上引入吡啶氮原子后, $\mathbf{A 2}$ 骨架中相应位置的扭转
二面角显著降低至 $5.3^{\circ}$. 相比化合物 $\mathbf{A 1}$, 从侧面观察发 现化合物 $\mathbf{A 2}$ 的骨架共平面性更好. 将 $\mathbf{A 1}$ 两两相连后, 所得二联体 BA1 分子骨架中的相应位置的扭转二面角 变化不大, 依然保持在 $11^{\circ}$ 和 $10.7^{\circ}$. 但是, BA1 中的两个 靛红单元之间的扭转二面角达 $33.9^{\circ}$, 因此 BA1 展现出 较大的骨架扭转. 在 $\mathbf{B A 2}$ 分子骨架上引入吡啶氮原子 后, 其骨架扭转二面角相比化合物 BA1 均明显降低(分 别为 $0.1^{\circ}, 8.0^{\circ}$ 和 $\left.8.0^{\circ}\right)$, 从侧面观察发现 BA2 的骨架共 平面性较 BA1 更好. 通常, 共平面性好的共轭骨架将减 少分子的无序堆积, 利于分子骨架的堆积排列, 进而获 得更高的载流子迁移率 ${ }^{[43]}$. 观察能级轨道分布情况可 知, 四个化合物均展现出相似离域的电子云分布情况, 其 HOMO 和 LUMO 能级的电子云近乎离域在整个分子 骨架，表明四个化合物分子具有较好的共轭结构 ${ }^{[22,44]}$. 由 DFT 计算得到的化合物 A1, A2, BA1 和 BA2 的 $\mathrm{HOMO} / \mathrm{LUMO}$ 能级分别为 $-6.03 /-3.14,-6.25$ / $-3.28,-5.95 /-3.45$ 和 $-6.02 /-3.60 \mathrm{eV}$. 对比计算能 级值可知: (1)相比 $\mathbf{A 1}$ 和 $\mathbf{A 2}$, 二联体化合物 (BA1 和 BA2)展现出更大共轭骨架、更强的电子亲和力以及更低 的 LUMO 能级值; (2)相比 A1 和 BA1, 吡啶氮原子的引 入增强了化合物 $\mathbf{A 2}$ 和 BA2 的电子亲和力和骨架共平面 性, 因而有效地降低了 $\mathbf{A 2}$ 和 BA2 的 HOMO 和 LUMO 能级值; (3)相比经典萗四甲酰二亚胺的计算 LUMO 能 级 $(-3.4 \mathrm{eV}$, 图 1), BA1 和 BA2 的计算 LUMO 能级值更 低, 因此可以预测化合物 BA1 和 BA2 为 $n$-型有机半导 体材料.

\section{4 吸收光谱}

采用吸收光谱仪测定了所得化合物 A1, A2, BA1 和 BA2 的溶液和薄膜紫外 - 可见吸收光谱, 相关的数据总 结在表 1 中. 图 $5 \mathrm{a}$ 给出了化合物在三氯甲烷溶液中的摩 尔消光系数和吸收峰型. 相比范醌二甲酰亚胺单元 (C13, 见辅助材料图 S3), $\mathbf{A 1}$ 和 $\mathbf{A 2}$ 在三氯甲烷中展现出 显著拓宽的吸收光谱, 且吸收峰型与经典的异靛青单元 (IID, 见辅助材料图 S3)非常相似. 在 300 450 nm 范围 内, $\mathbf{A 1}$ 和 $\mathbf{A 2}$ 展现出较强的吸收峰, 其最大摩尔摩尔消 光系数 $(\varepsilon)$ 分别达 $1.83 \times 10^{4}$ 和 $1.31 \times 10^{4} \mathrm{~mol} \cdot \mathrm{L}^{-1} \cdot \mathrm{cm}^{-1}$; 此外, A1 和 $\mathbf{A 2}$ 的最大吸收边带可以拓宽至 630 和 610 $\mathrm{nm}$ 处. 这些结果表明, $\mathbf{A 1}$ 和 $\mathbf{A 2}$ 是一类具有宽吸收的受 体单元, 可推广设计更多的 A-A 型和 A-D-A 型近红外 光捕获能力的有机半导体材料, 并在非富勒烯太阳能电 池和电子传输型有机场效应晶体管等领域中具有潜在 的应用前景.

相比 $\mathbf{A 1}$ 和 $\mathbf{A 2}$ 的溶液吸收, 二联体化合物 BA1 和 BA2 在 300 700 nm 范围内展现出更强的光捕获能力, 这归因于二联体化合物拥有更大的共轭骨架和更强的 


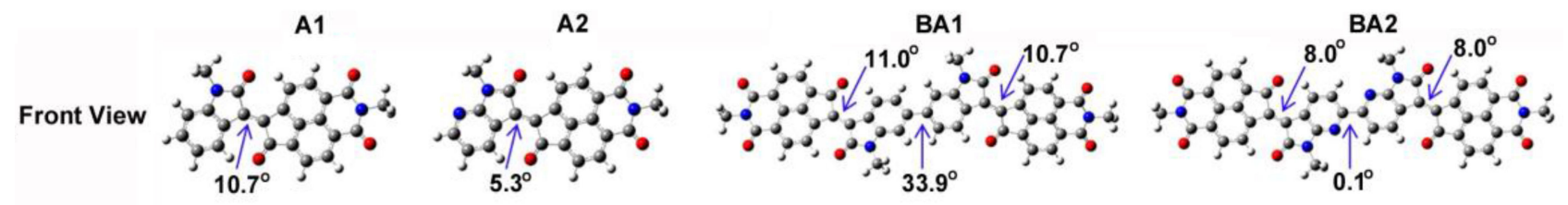

Side View $20 \%$,
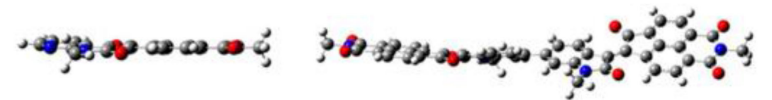

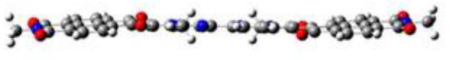

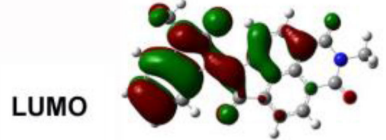

LUMO $=-\mathbf{3 . 1 4} \mathrm{eV}$

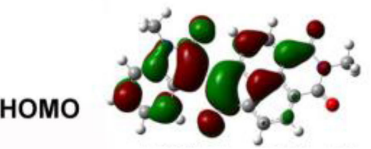

HOMO $=-6.03 \mathrm{eV}$

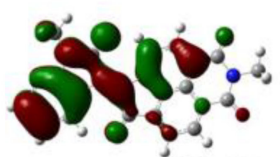

LUMO $=-3.28 \mathrm{eV}$

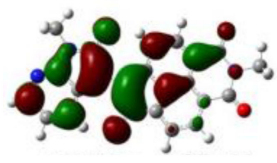

HOMO $=-6.25 \mathrm{eV}$

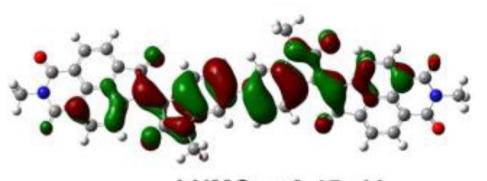

LUMO $=-3.45 \mathrm{eV}$

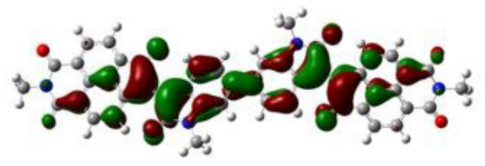

HOMO $=-5.95 \mathrm{eV}$

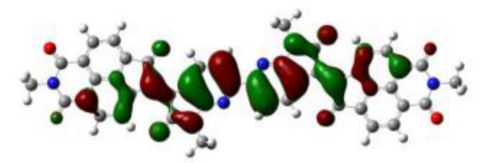

LUMO $=-3.60 \mathrm{eV}$

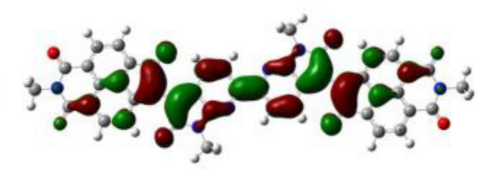

HOMO $=-6.02 \mathrm{eV}$

图 4 DFT 优化得到的分子构型(a)、前线分子轨道分布和 HOMO/LUMO 能级(b)

Figure 4 Optimized molecular structures (a), frontier molecular orbitals (b) and HOMO/LUMO energy levels obtained by DFT calculations

表 1 化合物 A1, A2, BA1 和 BA2 的光谱、电化学和 DFT 理论计算数据

Table 1 Optical, electrochemical and DFT-calculated data for compounds A1, A2, BA1 and BA2

\begin{tabular}{|c|c|c|c|c|c|c|c|c|c|}
\hline \multirow{2}{*}{ Compd. } & \multicolumn{2}{|c|}{$\lambda_{\max } / \mathrm{nm}$} & \multirow{2}{*}{$\lambda_{\text {edge }}$} & \multirow{2}{*}{$E_{\mathrm{g}}^{\mathrm{opt} a} / \mathrm{eV}$} & \multirow{2}{*}{$E_{\text {red }}^{\text {onset }} / \mathrm{eV}$} & \multirow{2}{*}{$E_{\mathrm{LUMO}}^{b} / \mathrm{eV}$} & \multirow{2}{*}{$E_{\mathrm{HOMO}}{ }^{c} / \mathrm{eV}$} & \multirow{2}{*}{$E_{\mathrm{LUMO}}{ }^{d} / \mathrm{eV}$} & \multirow{2}{*}{$E_{\mathrm{HOMO}}{ }^{d} / \mathrm{eV}$} \\
\hline & Sol. & Film & & & & & & & \\
\hline $\mathbf{A 1}$ & 371,417 & 370 & 650 & 1.91 & -0.46 & -3.96 & -5.87 & -3.14 & -6.03 \\
\hline $\mathbf{A 2}$ & $337,406,490$ & $330,414,500$ & 630 & 1.97 & -0.39 & -4.03 & -6.0 & -3.28 & -6.25 \\
\hline BA1 & 438,558 & 452,605 & 716 & 1.73 & -0.45 & -3.97 & -5.70 & -3.45 & -5.95 \\
\hline BA2 & $373,428,570$ & $358,577,626$ & 680 & 1.82 & -0.34 & -4.08 & -5.90 & -3.60 & -6.02 \\
\hline
\end{tabular}

分子内电荷转移作用 ${ }^{[44]}$. 在三氯甲烷溶液中, BA1 展现 出明显的双带吸收, 其溶液呈现为深褐色(图 5c). 而且, BA1 在 438 和 $560 \mathrm{~nm}$ 处分别有一个强的吸收顶峰和一 个相对弱的吸收肩峰, 对应的 $\varepsilon$ 分别达 $3.49 \times 10^{4}$ 和 $2.05 \times 10^{4} \mathrm{~mol} \cdot \mathrm{L}^{-1} \cdot \mathrm{cm}^{-1}$. 与 BA1 不同, BA2 在 $300 \sim 700$ $\mathrm{nm}$ 范围内展现四重吸收顶峰, 其溶液呈现为深紫色(图 5c). 相比 BA1, BA2 在 512 700 nm 处展现出更强的光 捕获能力, 并且在电荷转移吸收顶峰位置 $(570 \mathrm{~nm})$ 处的 $\varepsilon$ 高达 $3.67 \times 10^{4} \mathrm{~mol} \cdot \mathrm{L}^{-1} \cdot \mathrm{cm}^{-1}$. 结合 DFT 理论计算结果 分析: 相比骨架扭曲的 BA1, 引入吡啶氮原子增强了 BA2 的骨架平面性和分子内电荷转移作用, 因而提高了 BA2 的在可见光区的光捕获能力.

图 $5 \mathrm{~b}$ 和 $5 \mathrm{c}$ 给出了所得化合物的溶液和薄膜吸收光 谱的归一化对比图. 相比溶液吸收, 化合物 $\mathbf{A 1}, \mathbf{A 2}$, BA1 和 BA2 的薄膜末端吸收 $\left(\lambda_{\text {edge }}\right)$ 分别拓宽至 650, 630, 716 和 $680 \mathrm{~nm}$, 通过经验公式 $\left(E_{\mathrm{g}}^{\mathrm{opt}}=1240 / \lambda_{\text {edge }}\right)$ 换算得 到的光学带隙值分别为 $1.91,1.97,1.73$ 和 $1.82 \mathrm{eV}$. 由图 $5 \mathrm{c}$ 可知, BA1 薄膜在长波方向处的分子内电荷转移吸收
顶峰位于 $605 \mathrm{~nm}$, 相比溶液吸收顶峰红移了 $47 \mathrm{~nm}$. 此 外, BA2 薄膜在 500 700 nm 处的吸收顶峰变成两个 $(577$ 和 $626 \mathrm{~nm})$, 相比溶液吸收顶峰 $(570 \mathrm{~nm})$ 分别红移 了 7 和 $56 \mathrm{~nm}$. 这些红移现象表明, BA1 和 BA2 的固态 薄膜都存在强的分子间相互作用和有序 $J$-型聚集 ${ }^{[43-44]}$.

\section{5 电化学能级}

采用循环伏安法 $(\mathrm{CV})$ 测定了所得化合物 $\mathbf{A 1}, \mathbf{A} 2$, BA1 和 BA2 的电化学能级, 相关的数据总结在表 1 中. 从图 6 所示 $\mathrm{CV}$ 曲线可知, $\mathbf{A 1}$ 和 $\mathbf{A 2}$ 第一起始还原电位 $\left(E_{\text {red }}^{\text {onset }}\right)$ 分别处在 -0.46 和 $-0.39 \mathrm{~V}$ 处, 根据经验公式 $\left[E_{\mathrm{LUMO}}=-\left(4.42+E_{\mathrm{red}}^{\mathrm{onset}}\right) \mathrm{eV}\right]^{[42]}$ 计算得到的 LUMO 能级 值分别为 -3.96 和 $-4.03 \mathrm{eV}$. 进一步, 我们结合光谱光 学带隙 $\left(E_{\mathrm{g}}^{\mathrm{opt}}\right)$ 和经验公式 $\left[E_{\mathrm{HOMO}}=\left(E_{\mathrm{LUMO}}-E_{\mathrm{g}}^{\mathrm{opt}}\right) \mathrm{eV}\right]^{[42]}$, 间接计算出了化合物 $\mathbf{A 1}$ 和 $\mathbf{A 2}$ 的 HOMO 能级值(分别 为 -5.87 和 $-6.0 \mathrm{eV})$. 相比经典的异靛青单元的能级测 定值 $\left(E_{\mathrm{HOMO}}=-5.81 \mathrm{eV}\right.$ 和 $E_{\mathrm{LUMO}}=-3.71 \mathrm{eV}$, 见图 $\left.\mathrm{S} 3\right)$, $\mathbf{A 1}$ 和 $\mathbf{A 2}$ 的拥有更低的 HOMO/LUMO 能级值, 进一步 证实 $\mathrm{A1}$ 和 $\mathrm{A2}$ 为一类具有强缺电能力的受体单元. 观察 

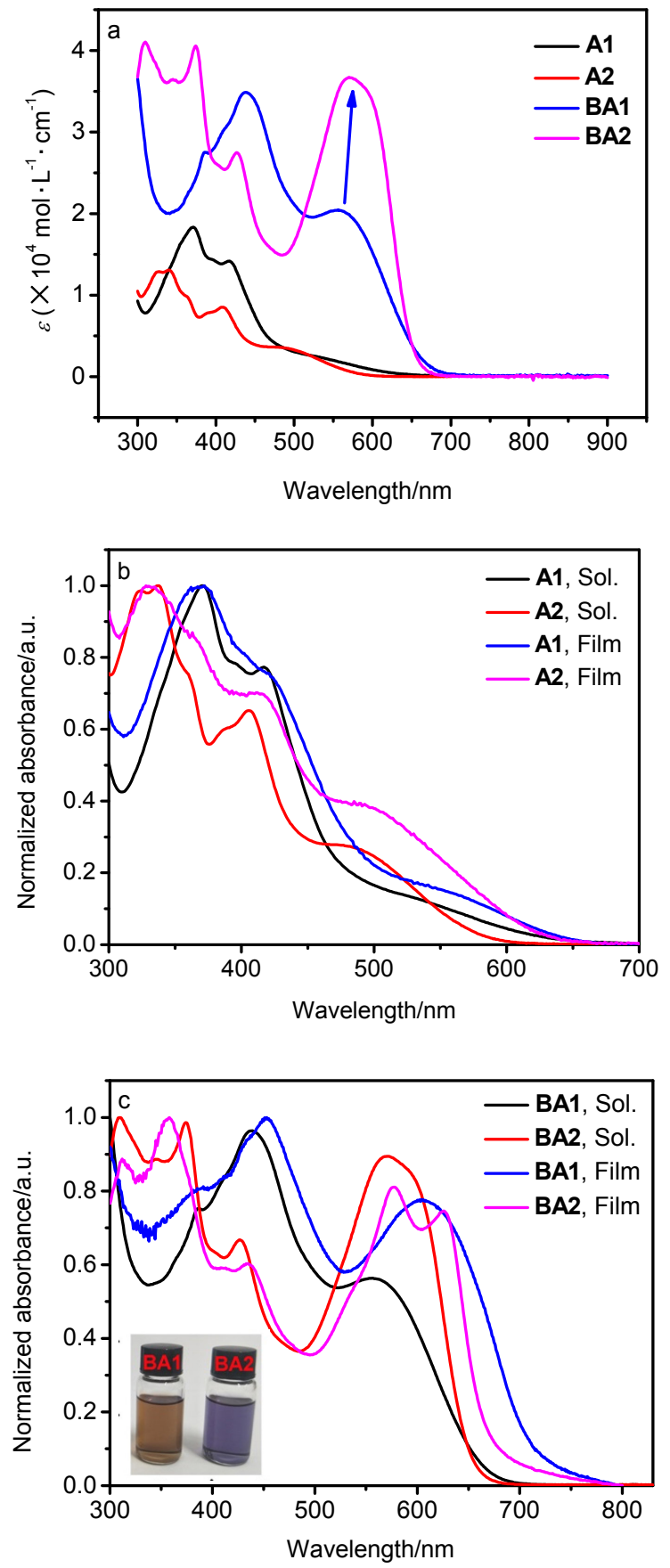

图 5 (a)所得化合物在三氯甲烷稀溶液中的摩尔消光系数, (b) $\mathrm{A1}$ 和 $\mathrm{A2}$ 在三氯甲烷稀溶液中和固态薄膜的吸收光谱, 以及 (c) BA1 和 BA2 在三氯甲烷稀溶液中和固态薄膜的吸收光谱 Figure 5 (a) Molar extinction coefficient of the as-synthesized compounds in chloroform solution, (b) absorption spectra of A1 and $\mathbf{A 2}$ in chloroform solution and solid-state film, and (c) absorption spectra of BA1 and BA2 in chloroform solution and solid-state film

$\mathrm{CV}$ 曲线发现，二联体化合物(BA1 和 BA2)在负电位方 向均展现出强而且可逆的氧化还原峰, 而在正电位方向 并为发现明显的氧化还原峰, 这些电化学特征行为揭示 BA1 和 BA2 为单极性 $n$-型有机半导体材料 ${ }^{[44]}$. 化合物
BA1 和 BA2 的 $E_{\text {red }}^{\text {onset }}$ 分别在 -0.45 和 $-0.34 \mathrm{~V}$ 处, 对应 的 LUMO 能级值分别为 -3.97 和 $-4.08 \mathrm{eV}$. 进一步，我 们间接换算得到了 BA1 和 BA2 的 HOMO 能级值, 分别 为 -5.70 和 $-5.90 \mathrm{eV}$. 对比以上所得能级值可知: 吡啶 氮原子的引入增强了 $\mathrm{A} 2$ 和 $\mathrm{BA} 2$ 的电子亲和力, 因而获 得较 A1 和 BA1 更低的 HOMO 和 LUMO 能级值, 这些 能级变化趋势与理论预测相一致.

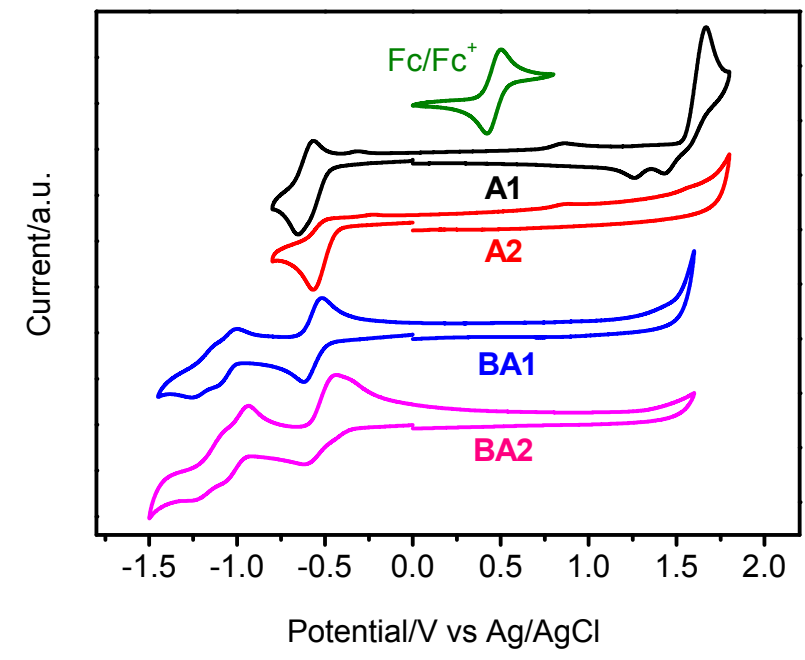

图 6 所得化合物在二氯甲烷溶液中的循环伏安曲线 Figure 6 Cyclic voltammogram curves of the compounds in dichloromethane solution

\section{6 载流子传输性能}

以上研究证实二联体化合物 BA1 和 BA2 具有好的 溶解性、优异的结构对称性、大的骨架共轭、理想的能 级结构以及强的电子亲和力等优势. 因此, 选取 BA1 和 BA2 为有机场效应晶体管器件的活性层材料, 进一步评 估了两个化合物的载流子传输性能, 具体器件制备程序 如下：(1)在氮气箱中, 采用溶液旋涂技术在十八烷基三 氯硅烷修饰的 $\mathrm{SiO}_{2} / \mathrm{Si}$ 衬底上制备一层厚度为 $50 \mathrm{~nm}$ 的 化合物 BA1 或 BA2 的薄膜; (2)将薄膜衬底置于 $120{ }^{\circ} \mathrm{C}$ 的热台上，退火处理 $10 \mathrm{~min}$; (3)利用掩模板沉积技术, 在活性层薄膜表面蒸镀厚度为 $50 \mathrm{~nm}$ 的铝源/漏电极. 在 氮气箱中, 采用半导体测试仪测定化合物 BA1 和 BA2 的载流子传输性能. 图 7 和辅助材料中图 S5 分别给出了 化合物 BA1 和 BA2 薄膜 OFET 器件的转移和输出特性 曲线. 当施加 $80 \mathrm{~V}$ 的源漏电压时, 两个化合物的薄膜 OFET 器件均展现出明显的单极性 $n$-型半导体特性, 其 电子迁移率分别达 $1.64 \times 10^{-3}$ 和 $2.52 \times 10^{-3} \mathrm{~cm}^{2} \cdot \mathrm{V}^{-1}$. $\mathrm{s}^{-1}$, 开关电流比均达到 $10^{3}$ 以上. 我们分析, 导致两个 化合物载流子迁移率差异的可能原因如下: 由理论计算 和能级值可以看出, 吡啶氮原子的引入增强了化合物 BA2 的骨架共平面性和电子亲和力, 因而获得了相比化 
合物 BA1 更高的电子迁移率. 尽管 BA1 和 BA2 的电子 迁移率相比文献报道经典 $n$-型有机半导体材料的要低, 但他们依然具备一些独特的优势(譬如宽的光谱响应范 围, 高的电子亲和力, 合适的 HOMO/LUMO 能级以及 极好的溶解性等), 因而 BA1 和 BA2 有望在非富勒烯太 阳能电池器件应用中具备一定的前景.

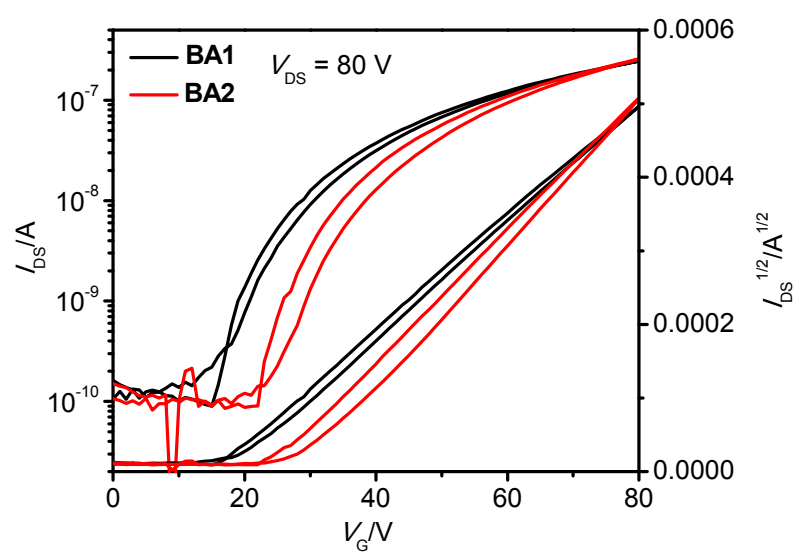

图 7 基于化合物 BA1 和 BA2 薄膜构造的 OFET 器件的转移 特性曲线

Figure 7 Transfer curves of the OFET devices fabricated from compounds BA1 or BA2

\section{2 结论}

本工作将靛红和氮杂靛红衍生物单元分别与范醌 二甲酰亚胺进行 Knoevenagel 缩合, 成功制备了两个新 型异靛青衍生物受体单元 $\mathbf{A 1}$ 和 $\mathbf{A 2}$. 相比经典的异靛青 受体, $\mathbf{A 1}$ 和 $\mathbf{A 2}$ 展现出相似的吸收光谱特征、更强的电 子亲和力以及更低的 LUMO 能级值 $(c a .-4.0 \mathrm{eV})$, 这 些结果证实 $\mathbf{A} 1$ 和 $\mathbf{A} 2$ 是一类骨架共轭大、吸电子能力 强的 $n$-型有机半导体材料构建模块. 进一步, 本工作运 用 “A-A 双受体策略”, 开发了两个含 $\mathbf{A} 1$ 和 A2 受体单 元的二联体共轭分子 BA1 和 BA2, 并探讨了吡啶氮原 子对目标共轭分子的骨架结构、吸收光谱、HOMO/ LUMO 能级以及电子迁移率的影响. 研究发现: (1)将两 个不对称的 $\mathrm{A} 1$ 和 $\mathrm{A} 2$ 受体两两相连, 不仅有效地提高了 BA1 和 BA2 的骨架共轭和分子对称性, 而且显著提升 了 BA1 和 BA2 在整个紫外-可见光区的光捕获能力; (2) 相比 $\mathrm{A1}$ 和 $\mathrm{BA2}$ 受体, 含吡啶氮原子的 $\mathrm{A} 2$ 和 $\mathrm{BA2}$ 拥有 更好的骨架共平面性、更强电子亲和力以及更低 HOMO 和 LUMO 能级值. 为评估化合物的载流子传输性能, 我 们制备了基于 BA1 和 BA2 薄膜的 $n$-型场效应晶体管器 件, 测得其电子迁移率分别为 $1.64 \times 10^{-3}$ 和 $2.52 \times 10^{-3}$ $\mathrm{cm}^{2} \cdot \mathrm{V}^{-1} \cdot \mathrm{s}^{-1}$. 以上研究结果揭示, A1 和 $\mathrm{A} 2$ 是一类具有 吸收光谱宽、骨架共轭大以及电子亲和力强的新型电子 受体单元, 可进一步推广用于开发 A-D-A 型近红外光 捕获能力的有机电子传输材料, 在非富勒烯太阳能电池
和电子传输型有机场效应晶体管等领域中具有广阔的 应用前景.

\section{3 实验部分}

\section{1 仪器与试剂}

核磁共振 ${ }^{1} \mathrm{H}$ NMR $/{ }^{13} \mathrm{C}$ NMR 采用 Bruker AVANCE 400 测定, 所用溶剂和内标分别为気代三氯甲烷 $\left(\mathrm{CDCl}_{3}\right)$ 和四甲基硅烷(TMS). 质谱(MS)采用 MALDI-TOF 由 Bruker Biflex III MALDI-TOF 质谱仪测定. HRMS (MALDI)由 IonSpec 4.7 Tesla Fourier Transform 型质谱 仪测定. 紫外-可见吸收光谱由 Perkin-Elmer Lamada 25 分光光度计测得. 采用密度泛函理论计算了模型分子的 结构和前线轨道能级, 所选机组为 B3LYP/6-31G(d). 在 氮气保护下，采用热重分析仪(TGA, DSC-Q10)和示差 扫描量热分析仪(DSC, Perkin-Elmer TGA-7)测定了化合 物的热稳定性和热力学相转变, 其升温速度为 $10{ }^{\circ} \mathrm{C} \cdot$ $\mathrm{min}^{-1}$. 采用电化学工作站(CHI660E, Chenhua, Shanghai)在 $100 \mathrm{mV} \cdot \mathrm{s}^{-1}$ 的扫描速度下测定化合物的循环伏安 曲线 $(\mathrm{CV})$. 所用三电极体系电化学池包括玻璃碳工作电 极、 $\mathrm{Ag} / \mathrm{AgCl}\left(0.01 \mathrm{~mol} \cdot \mathrm{L}^{-1} \mathrm{KCl}\right)$ 参比电极、铂丝对电极、 $0.1 \mathrm{~mol} \cdot \mathrm{L}^{-1}$ 的四丁基六氟磷酸铵二氯甲烷溶液电解质. 所用内标为二茂铁 $(0.38 \mathrm{~V}$ vs. $\mathrm{Ag} / \mathrm{AgCl})$. 采用 Angilent B2912A 测定了薄膜 OFET 器件的转移和输出特性曲线.

化合物 C1、C2、C5、C6 和六甲基二锡烷等试剂 购自上海毕得医药科技有限公司，氯铬酸吡啶、对甲基 苯磺酸、氢化钠 $(\mathrm{NaH})$ 、三氯化铝、水合肼和五氧化二 磷购自武汉长成化成科技发展有限公司，其它常用溶剂 和试剂均购自国药集团化学试剂有限公司.

\section{2 实验方法}

化合物 $\mathbf{C 3}^{[37]} 、 \mathbf{C 4}^{[38]} 、 \mathbf{C 7}^{[39]} 、 \mathbf{C}^{[39]} 、 \mathbf{C 1 1}^{[37]} 、 \mathbf{C 1 3}^{[40]}$

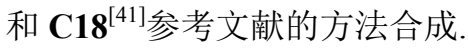

3.2.1 6-溴-1-(2-乙基已基)-1H-吡咯并 [2,3- $b]$ 吡啶 (C8) 的合成

在 $\mathrm{N}_{2}$ 保护下, 向 $100 \mathrm{~mL}$ 的三口瓶中加入 $\mathrm{NaH}(0.74$ $\mathrm{g}, 30.83 \mathrm{mmol}$ )和 $10 \mathrm{~mL}$ 的 $N, N^{\prime}$-二甲酰胺 $(\mathrm{DMF})$ 后, 在 $0{ }^{\circ} \mathrm{C}$ 下缓慢加入 6-溴-7-氮杂吲哚(3.0 g, $\left.15.23 \mathrm{mmol}\right)$. 搅拌 $20 \mathrm{~min}$ 后加入溴代异辛烷 $(4.4 \mathrm{~g}, 22.78 \mathrm{mmol})$, 在室 温下反应过夜. 采用乙酸乙酯和饱和食盐水萃取, 无水 硫酸镁干燥，过滤，溶剂减压旋干，所得粗产品采用硅 胶色谱柱提纯(洗脱剂为石油醚)得 $4.14 \mathrm{~g}$ 淡黄色粘稠液 体(产率 88\%). ${ }^{1} \mathrm{H}$ NMR $\left(400 \mathrm{MHz}, \mathrm{CDCl}_{3}\right) \delta: 7.73(\mathrm{~d}$, $J=8.0 \mathrm{~Hz}, 1 \mathrm{H}), 7.17(\mathrm{~d}, J=8.0 \mathrm{~Hz}, 1 \mathrm{H}), 7.15$ (d, $J=3.2$ $\mathrm{Hz}, 1 \mathrm{H}), 6.43$ (d, $J=3.6 \mathrm{~Hz}, 1 \mathrm{H}), 4.15$ (d, $J=7.2 \mathrm{~Hz}, 2 \mathrm{H})$, $1.94 \sim 1.89(\mathrm{~m}, 1 \mathrm{H}), 1.33 \sim 1.20(\mathrm{~m}, 8 \mathrm{H}), 0.92 \sim 0.85(\mathrm{~m}$, $6 \mathrm{H}) ;{ }^{13} \mathrm{C}$ NMR (100 MHz, $\left.\mathrm{CDCl}_{3}\right) \delta: 147.5,134.5,130.7$, 
128.3, 119.1, 119.0, 99.7, 48.0, 40.2, 30.4, 28.5, 23.9, 23.0, 14.1, 10.6. HRMS (ESI) calcd for $\mathrm{C}_{15} \mathrm{H}_{21} \mathrm{BrN}_{2} 308.0887$, found 308.0888 .

3.2.2 6-溴-1-(2-乙基已基)- $1 H$-吡咯并 [2,3- $b]$ 吡啶$2,3-$ 二酮 $(\mathbf{C 1 0})$ 的合成

向 $100 \mathrm{~mL}$ 的三口瓶中加入 $1.5 \mathrm{~g}$ 硅胶粉、氯铬酸吡 啶盐(6.3 g, $29.22 \mathrm{mmol}) 、$ 化合物 $\mathbf{C 8}(2.7 \mathrm{~g}, 8.73 \mathrm{mmol}) 、$ $20 \mathrm{~mL}$ 的乙腈和 $20 \mathrm{~mL}$ 的二氯甲烷, 最后加入 $10 \mathrm{mg}$ 的 三氯化铝. 在 $\mathrm{N}_{2}$ 保护下, 回流反应 $3 \mathrm{~h}$ 后, 溶剂减压旋 干后, 所得粗产品采用硅胶色谱柱提纯[洗脱剂为 $V($ 石 油醚)： $V$ (二氯甲烷) $=1 ： 1$ 得 $1.42 \mathrm{~g}$ 黄色粉末(产率 48\%). m.p. 94 96 ${ }^{\circ} \mathrm{C}$; ${ }^{1} \mathrm{H}$ NMR (400 MHz, $\left.\mathrm{CDCl}_{3}\right) \delta$ : 7.64 (d, $J=8.0 \mathrm{~Hz}, 1 \mathrm{H}), 7.27$ (d, $J=4.4 \mathrm{~Hz}, 1 \mathrm{H}), 3.72$ (d, $J=8.0 \mathrm{~Hz}, 2 \mathrm{H}), 1.97 \sim 1.92(\mathrm{~m}, 1 \mathrm{H}), 1.41 \sim 1.30(\mathrm{~m}, 8 \mathrm{H})$, $0.95 \sim 0.88(\mathrm{~m}, 6 \mathrm{H}) ;{ }^{13} \mathrm{C} \mathrm{NMR}\left(100 \mathrm{MHz}, \mathrm{CDCl}_{3}\right) \delta$ : $180.9,164.5,158.5,150.2,134.0,123.3,110.2$, 43.4, 37.4, 30.4, 28.3, 23.9, 23.0, 14.1, 10.4. HRMS (ESI) calcd for $\mathrm{C}_{15} \mathrm{H}_{19} \mathrm{BrN}_{2} \mathrm{O}_{2} 338.0630$, found 338.0634.

3.2.3 1-乙基- $1 H$-吡咯并 $[2,3-b]$ 吡啶-2(3H)-酮(C12) 的合成

向 $100 \mathrm{~mL}$ 的三口瓶中加入化合物 C9 (200 mg, 1.14 $\mathrm{mmol}) 、 10 \mathrm{~mL}$ 的水合肼和 $10 \mathrm{~mL}$ 的 1,4-二氧六环溶剂. 在 $\mathrm{N}_{2}$ 保护下, $120{ }^{\circ} \mathrm{C}$ 下反应 $2 \mathrm{~d}$. 冷却至室温后, 采用二 氯甲烷和饱和食盐水萃取, 无水硫酸镁干燥. 经无水硫 酸镁干燥后过滤, 旋干溶剂得粗产品, 采用硅胶色谱柱 提纯 [洗脱剂为 $V$ (石油醚) $: V($ 二氯甲烷 $)=1 ： 1$ ] 得 101.3 $\mathrm{mg}$ 无色粘稠液体 C12, 产率 55\%. ${ }^{1} \mathrm{H}$ NMR (400 MHz, $\left.\mathrm{CDCl}_{3}\right) \delta: 8.17(\mathrm{~d}, J=4.8 \mathrm{~Hz}, 1 \mathrm{H}), 7.47$ (dd, $J=8.0,4.0$ $\mathrm{Hz}, 1 \mathrm{H}), 6.93(\mathrm{dd}, J=8.0,4.0 \mathrm{~Hz}, 1 \mathrm{H}), 3.91 \sim 3.86$ (q, 2H), 3.51 (s, 2H), 1.30 (t, $J=8.0 \mathrm{~Hz}, 3 \mathrm{H}) ;{ }^{13} \mathrm{C}$ NMR $(100$ $\left.\mathrm{MHz} \mathrm{CDCl}_{3}\right) \delta: 174.1,157.9,146.8,131.6,119.3,117.8$, 34.9, 34.1, 13.1. HRMS (ESI) calcd for $\mathrm{C}_{9} \mathrm{H}_{10} \mathrm{~N}_{2} \mathrm{O}$ 162.0793, found 162.0791.

\section{2 .4 化合物 $\mathbf{A 1}$ 的合成}

将化合物 C11 (86 mg, $0.49 \mathrm{mmol}) 、$ 化合物 C13 (201.6 mg, $0.49 \mathrm{mmol}$ )、 $1 \mathrm{mg}$ 的 $\mathrm{P}_{2} \mathrm{O}_{5}$ 、对甲基苯磺酸(10 $\mathrm{mg}, 0.058 \mathrm{mmol}$ )和 $10 \mathrm{~mL}$ 的甲苯加入到 $100 \mathrm{~mL}$ 的三口 瓶中. 在 $\mathrm{N}_{2}$ 保护下, 置于 $120{ }^{\circ} \mathrm{C}$ 油浴锅搅拌 $6 \mathrm{~h}$. 冷却 至室温, 用二氯甲烷和饱和食盐水萃取. 经无水硫酸镁 干燥后过滤, 旋干溶剂得粗产品, 采用硅胶色谱柱提纯 [洗脱剂为 $V$ (石油醚) $: V($ 二氯甲烷 $)=1 ： 1$ ] 得 $135.9 \mathrm{mg}$ 黄棕色粉末 A1, 产率 50\%. m.p. > $300{ }^{\circ} \mathrm{C} ;{ }^{1} \mathrm{H}$ NMR (400 $\left.\mathrm{MHz} \mathrm{CDCl}_{3}\right) \delta: 9.81(\mathrm{~d}, J=8.0 \mathrm{~Hz}, 1 \mathrm{H}), 9.08$ (d, $J=8.0$ $\mathrm{Hz}, 1 \mathrm{H}), 8.75$ (dd, $J=12.0,8.0 \mathrm{~Hz}, 2 \mathrm{H}), 8.27$ (d, $J=8.0$ $\mathrm{Hz}, 1 \mathrm{H}), 7.53 \sim 7.45(\mathrm{~m}, 2 \mathrm{H}), 7.36(\mathrm{~d}, J=8.0 \mathrm{~Hz}, 2 \mathrm{H})$, $7.15(\mathrm{t}, J=8.0 \mathrm{~Hz}, 1 \mathrm{H}), 6.88(\mathrm{~d}, J=8.0 \mathrm{~Hz}, 1 \mathrm{H}), 3.91(\mathrm{q}$, $J=8.0 \mathrm{~Hz}, 2 \mathrm{H}), 2.80 \sim 2.73(\mathrm{~m}, 2 \mathrm{H}), 1.37(\mathrm{t}, J=8.0 \mathrm{~Hz}$, $3 \mathrm{H}), 1.13$ (q, $J=4.0 \mathrm{~Hz}, 12 \mathrm{H}) ;{ }^{13} \mathrm{C} \mathrm{NMR}(100 \mathrm{MHz}$, $\left.\mathrm{CDCl}_{3}\right) \delta: 192.4,167.7,163.6,163.4,145.7,145.3,135.8$, $133.8,133.6,131.8,130.7,130.6,129.7,129.0,126.2$, $125.9,124.1,122.6,122.3,122.0,121.5,108.3,34.9,29.2$, 24.0, 12.63. HRMS (MALDI) calcd for $\mathrm{C}_{36} \mathrm{H}_{31} \mathrm{~N}_{2} \mathrm{O}_{4}[\mathrm{M}+$ $\mathrm{H}]^{+}$555.2278, found 555.2275.

\section{2 .5 化合物 $\mathbf{A 2}$ 的合成}

将化合物 $\mathbf{C 1 2}(86.3 \mathrm{mg}, 0.49 \mathrm{mmol}) 、$ 化合物 C13 (201.6 mg, $0.49 \mathrm{mmol}$ )、 $1 \mathrm{mg}$ 的 $\mathrm{P}_{2} \mathrm{O}_{5}$ 、对甲基苯磺酸(10 $\mathrm{mg}, 0.058 \mathrm{mmol}$ )以及 $10 \mathrm{~mL}$ 的甲苯置于 $100 \mathrm{~mL}$ 的三口 瓶中. 在 $\mathrm{N}_{2}$ 保护下, 于 $120{ }^{\circ} \mathrm{C}$ 油浴锅中搅拌 $6 \mathrm{~h}$. 冷却 至室温后，加水淬灭反应，用二氯甲烷和饱和食盐萃取， 无水硫酸镁干燥, 过滤, 旋干溶剂得粗产品. 采用硅胶 色谱柱提纯粗产品 $\mathbf{A} 2$ [洗脱剂为 $V$ (石油醚)： $V$ (二氯甲 烷 $)=1 ： 1$ ]得 $149.7 \mathrm{mg}$ 红棕色粉末, 产率 55\%. m.p.> $300{ }^{\circ} \mathrm{C} ;{ }^{1} \mathrm{H}$ NMR (400 MHz, $\left.\mathrm{CDCl}_{3}\right) \delta: 9.87(\mathrm{~d}, J=8.0 \mathrm{~Hz}$, $1 \mathrm{H}), 9.36(\mathrm{~d}, J=8.0 \mathrm{~Hz}, 1 \mathrm{H}), 8.77$ (dd, $J=8.0,4.0 \mathrm{~Hz}$, $2 \mathrm{H}), 8.32$ (d, $J=4.0 \mathrm{~Hz}, 1 \mathrm{H}), 8.28$ (d, $J=8.0 \mathrm{~Hz}, 1 \mathrm{H}), 7.51$ (t, $J=8.0 \mathrm{~Hz}, 1 \mathrm{H}), 7.36(\mathrm{~d}, J=8.0 \mathrm{~Hz}, 1 \mathrm{H}), 7.10(\mathrm{dd}, J=$ $8.0,8.0 \mathrm{~Hz}, 1 \mathrm{H}), 4.05$ (q, $J=8.0 \mathrm{~Hz}, 2 \mathrm{H}), 2.79 \sim 2.73(\mathrm{~m}$, $2 \mathrm{H}), 1.41(\mathrm{t}, J=8.0 \mathrm{~Hz}, 3 \mathrm{H}), 1.18$ (q, $J=4.0 \mathrm{~Hz}, 12 \mathrm{H}) ;{ }^{13} \mathrm{C}$ NMR (100 MHz, $\left.\mathrm{CDCl}_{3}\right) \delta: 192.3,167.4,163.4,163.2$, $157.9,151.0,145.7,139.9,138.9,137.8,137.6,135.4$, $133.6,133.2$, 131.9, 130.6, 129.8, 129.5, 126.2, 126.1, 124.2, 122.7, 122.2, 118.6, 116.2, 34.4, 29.2, 24.0, 13.1. HRMS (MALDI) calcd $\mathrm{C}_{35} \mathrm{H}_{30} \mathrm{~N}_{3} \mathrm{O}_{4}[\mathrm{M}+\mathrm{H}]^{+}$556.2230, found 556.2228.

\subsection{6 化合物 C14 的合成}

向 $100 \mathrm{~mL}$ 的三口瓶中加入化合物 $\mathbf{C 4}$ (203.5 mg, $0.60 \mathrm{mmol}$ )、六甲基二锡烷 $(98.3 \mathrm{mg}, 0.30 \mathrm{mmol}$ )、双(三 苯基膦)二氯化钯 $(35 \mathrm{mg}, 0.05 \mathrm{mmol})$ 和 $10 \mathrm{~mL}$ 的甲苯. 在 $\mathrm{N}_{2}$ 保护下，回流反应 $3 \mathrm{~h}$ 后，减压旋干溶剂，所得粗 产品采用硅胶色谱柱提纯[洗脱剂为 $V$ (石油醚) : $V$ (二氯 甲烷 $)=1 ： 2$ ]得 $119.4 \mathrm{mg}$ 黄色粉末 C14, 产率 77\%. m.p. $180 \sim 184{ }^{\circ} \mathrm{C} ;{ }^{1} \mathrm{H}$ NMR (400 MHz, $\left.\mathrm{CDCl}_{3}\right) \delta: 7.73$ (d, $J=$ $8.0 \mathrm{~Hz}, 2 \mathrm{H}), 7.30(\mathrm{~d}, J=4.0 \mathrm{~Hz}, 2 \mathrm{H}), 7.01(\mathrm{~s}, 2 \mathrm{H}), 3.68(\mathrm{~d}$, $J=8.0 \mathrm{~Hz}, 4 \mathrm{H}), 1.84(\mathrm{~m}, 2 \mathrm{H}), 1.45 \sim 1.30(\mathrm{~m}, 16 \mathrm{H}), 0.96$ $(\mathrm{t}, J=8.0 \mathrm{~Hz}, 6 \mathrm{H}), 0.88(\mathrm{t}, J=8.0 \mathrm{~Hz}, 6 \mathrm{H}) ;{ }^{13} \mathrm{C} \mathrm{NMR}(100$ $\left.\mathrm{MHz} \mathrm{CDCl}_{3}\right) \delta: 182.9,158.5,152.1,149.6,126.0,122.8$, 117.6, 109.1, 44.5, 37.5, 30.6, 28.6, 24.0, 23.0, 14.1, 10.7 . HRMS (MALDI) calcd for $\mathrm{C}_{32} \mathrm{H}_{43} \mathrm{~N}_{2} \mathrm{O}_{4}[\mathrm{M}+\mathrm{H}]$ 519.3217, found 519.3215. 


\section{2 .4 化合物 $\mathbf{C 1 5}$ 的合成}

向 $100 \mathrm{~mL}$ 的三口瓶中加入化合物 $\mathbf{C 1 0}$ (203.5 mg, $0.60 \mathrm{mmol}$ )、六甲基二锡烷 $(98.3 \mathrm{mg}, 0.30 \mathrm{mmol}$ )、双(三 苯基膦)二氯化钯 $(35 \mathrm{mg}, 0.05 \mathrm{mmol}) 、 5 \mathrm{~mL}$ 的甲苯. 在 $\mathrm{N}_{2}$ 保护下, 回流反应 $3 \mathrm{~h}$ 后, 溶剂减压旋干, 所得粗产 品采用硅胶色谱柱提纯 [洗脱剂为 $V$ (石油醚)： $V$ (二氯甲 烷 $)=1$ : 2]得 $132.3 \mathrm{mg}$ 黄色粉末 C15, 产率 85\%. m.p. 199 $210{ }^{\circ} \mathrm{C} ;{ }^{1} \mathrm{H}$ NMR (400 MHz, $\left.\mathrm{CDCl}_{3}\right) \delta: 8.27$ (d, $J=$ $8.0 \mathrm{~Hz}, 2 \mathrm{H}), 7.99$ (d, $J=8.0 \mathrm{~Hz}, 2 \mathrm{H}), 3.85$ (d, $J=4.0 \mathrm{~Hz}$, $4 \mathrm{H}), 2.03 \sim 2.00(\mathrm{~m}, 2 \mathrm{H}), 1.46 \sim 1.28(\mathrm{~m}, 16 \mathrm{H}), 0.97(\mathrm{t}$, $J=8.0 \mathrm{~Hz}, 6 \mathrm{H}), 0.90(\mathrm{t}, J=8.0 \mathrm{~Hz}, 6 \mathrm{H}) ;{ }^{13} \mathrm{C}$ NMR $(100$ $\left.\mathrm{MHz}, \mathrm{CDCl}_{3}\right) \delta: 181.6,164.0,159.2,158.8,133.8,117.9$, 113.0, 43.3, 37.7, 30.7, 28.7, 24.1, 23.0, 14.2, 10.6. HRMS (MALDI) calcd for $\mathrm{C}_{30} \mathrm{H}_{41} \mathrm{~N}_{4} \mathrm{O}_{4}[\mathrm{M}+\mathrm{H}]^{+}$521.3122, found 521.3122 .

\subsection{5 化合物 C16 的合成}

向 $100 \mathrm{~mL}$ 的三口瓶中加入化合物 C14 (201.5 mg, $0.39 \mathrm{mmol}) 、 10 \mathrm{~mL}$ 的水合肼和 $10 \mathrm{~mL}$ 的 1,4-二氧六环 溶剂. 在 $\mathrm{N}_{2}$ 保护下于 $120{ }^{\circ} \mathrm{C}$ 的油浴锅中反应 $2 \mathrm{~d}$. 冷却 至室温后, 采用二氯甲烷和饱和食盐水萃取, 无水硫酸 镁干燥, 过滤, 溶剂减压旋干, 所得粗产品采用硅胶色 谱柱提纯 [洗脱剂为 $V$ (石油醚) $: V$ (二氯甲烷 $)=1: 1$ ] 得 $120 \mathrm{mg}$ 淡黄色粉末 C16, 产率 63\%. m.p. 99 101 ${ }^{\circ} \mathrm{C}$; ${ }^{1} \mathrm{H}$ NMR (400 MHz, $\left.\mathrm{CDCl}_{3}\right) \delta: 7.32(\mathrm{~d}, J=4.0 \mathrm{~Hz}, 2 \mathrm{H})$, $7.22(\mathrm{~d}, J=8.0 \mathrm{~Hz}, 2 \mathrm{H}), 6.98(\mathrm{~s}, 2 \mathrm{H}), 3.65(\mathrm{dd}, J=8.0 \mathrm{~Hz}$, $4 \mathrm{H}), 3.59(\mathrm{~s}, 4 \mathrm{H}), 1.87 \sim 1.84(\mathrm{~m}, 2 \mathrm{H}), 1.42 \sim 1.29(\mathrm{~m}$, $16 \mathrm{H}), 0.94(\mathrm{t}, J=8.0 \mathrm{~Hz}, 6 \mathrm{H}), 0.88(\mathrm{t}, J=8.0 \mathrm{~Hz}, 6 \mathrm{H}) ;{ }^{13} \mathrm{C}$ NMR $\left(100 \mathrm{MHz}, \mathrm{CDCl}_{3}\right) \delta: 182.9,158.5,152.1,149.6$, 126.0, 122.8, 117.6, 109.1, 44.5, 37.5, 30.6, 28.6, 24.0, 23.0, 14.1, 10.7. HRMS (MALDI) calcd for $\mathrm{C}_{32} \mathrm{H}_{45} \mathrm{~N}_{2} \mathrm{O}_{2}$ $[\mathrm{M}+\mathrm{H}]^{+}$489.3475, found 489.3474.

\subsection{6 化合物 $\mathbf{C 1 7}$ 的合成}

向 $100 \mathrm{~mL}$ 的三口瓶中加入化合物 C15 (202.3 mg, $0.39 \mathrm{mmol}$ )、 $10 \mathrm{~mL}$ 的水合肼和 $10 \mathrm{~mL}$ 的 1,4-二氧六环 溶剂. 在 $\mathrm{N}_{2}$ 保护下, 于 $120{ }^{\circ} \mathrm{C}$ 的油浴锅中反应 $2 \mathrm{~d}$. 冷 却至室温后, 采用二氯甲烷和饱和食盐水萃取, 无水硫 酸镁干燥, 过滤, 溶剂减压旋干, 所得采用硅胶色谱柱 提纯 [洗脱剂为 $V$ (石油醚) $: V($ 二氯甲烷 $)=1: 1$ ] 得 103.3 $\mathrm{mg}$ 淡黄色粉末 C17, 产率 54\%. m.p. $180 \sim 182{ }^{\circ} \mathrm{C} ;{ }^{1} \mathrm{H}$ NMR (400 MHz, $\left.\mathrm{CDCl}_{3}\right) \delta: 8.09(\mathrm{~d}, J=8.0 \mathrm{~Hz}, 2 \mathrm{H}), 7.57$ $(\mathrm{d}, J=8.0 \mathrm{~Hz}, 2 \mathrm{H}), 3.80(\mathrm{~d}, J=8.0 \mathrm{~Hz}, 4 \mathrm{H}), 3.58(\mathrm{~s}, 4 \mathrm{H})$, $2.06 \sim 2.03(\mathrm{~m}, 2 \mathrm{H}), 1.45 \sim 1.27(\mathrm{~m}, 16 \mathrm{H}), 0.95(\mathrm{t}, J=8.0$ $\mathrm{Hz}, 6 \mathrm{H}), 0.89$ (t, $J=8.0 \mathrm{~Hz}, 6 \mathrm{H}) ;{ }^{13} \mathrm{C}$ NMR $(100 \mathrm{MHz}$, $\left.\mathrm{CDCl}_{3}\right) \delta: 181.6,164.0,159.2,158.8,133.8,117.9,113.0$, $43.3,37.7,30.7,28.7,24.1,23.0,14.2,10.6$. HRMS
(MALDI) calcd for $\mathrm{C}_{30} \mathrm{H}_{43} \mathrm{~N}_{4} \mathrm{O}_{2}[\mathrm{M}+\mathrm{H}]^{+}$491.3380, found 491.3379 .

\section{2 .7 化合物 BA1 的合成}

将化合物 C16 (97.7 mg, $0.2 \mathrm{mmol}$ )、化合物 C18 (106.3 mg, $0.2 \mathrm{mmol}$ )、 $1 \mathrm{mg}$ 的 $\mathrm{P}_{2} \mathrm{O}_{5}$ 、对甲基苯磺酸( $5 \mathrm{mg}$, $0.029 \mathrm{mmol}$ )和 $10 \mathrm{~mL}$ 的甲苯置于 $100 \mathrm{~mL}$ 的三口瓶中. 在 $\mathrm{N}_{2}$ 保护下, 于 $120{ }^{\circ} \mathrm{C}$ 的油浴锅中搅拌 $12 \mathrm{~h}$. 冷却至室 温, 采用二氯甲烷和饱和食盐萃取, 经无水硫酸镁干燥 后过滤, 旋干溶剂得粗产品. 进一步, 采用硅胶色谱柱 提纯 [洗脱剂为 $V$ (石油醚) $: V$ (二氯甲烷 $)=1 ： 1$ ] 得 118.3 $\mathrm{mg}$ 棕色粉末 BA1, 产率 39\%. m.p.>300 ${ }^{\circ} \mathrm{C} ;{ }^{1} \mathrm{H} \mathrm{NMR}$ $\left(400 \mathrm{MHz}, \mathrm{CDCl}_{3}\right) \delta: 9.68(\mathrm{~d}, J=8.0 \mathrm{~Hz}, 2 \mathrm{H}), 9.06(\mathrm{~d}, J=$ $8.0 \mathrm{~Hz}, 2 \mathrm{H}), 8.67$ (d, $J=8.0 \mathrm{~Hz}, 2 \mathrm{H}), 8.61(\mathrm{~d}, J=8.0 \mathrm{~Hz}$, $2 \mathrm{H}), 8.20(\mathrm{~d}, J=8.0 \mathrm{~Hz}, 2 \mathrm{H}), 7.31(\mathrm{~d}, J=8.0 \mathrm{~Hz}, 2 \mathrm{H}), 7.02$ (s, 2H), 4.01 (br, 4H), 3.82 (d, J=8.0 Hz, 4H), 1.94 (br, $4 \mathrm{H}), 1.51 \sim 1.21(\mathrm{~m}, 80 \mathrm{H}), 1.04(\mathrm{t}, J=8.0 \mathrm{~Hz}, 6 \mathrm{H}), 0.93(\mathrm{t}$, $J=8.0 \mathrm{~Hz}, 6 \mathrm{H}), 0.88 \sim 0.83(\mathrm{~m}, 12 \mathrm{H}) ;{ }^{13} \mathrm{C}$ NMR $(100$ $\left.\mathrm{MHz} \mathrm{CDCl}_{3}\right) \delta: 192.4,168.4,163.6,163.4,153.4,146.6$, $144.4,142.6,139.2,138.0,137.8,135.3,134.1,132.9$, $131.2,130.8,129.1,125.9,125.3,122.3,121.9,121.5$, 120.6, 106.5, 44.6, 44.4, 37.9, 36.6, 31.9, 31.9, 31.7, 30.1, 29.7, 29.7, 29.6, 29.6, 29.4, 29.3, 26.5, 23.2, 22.7, 22.7, 14.1, 10.9. HRMS (MALDI) calcd for $\mathrm{C}_{100} \mathrm{H}_{131} \mathrm{~N}_{4} \mathrm{O}_{8}[\mathrm{M}+$ $\mathrm{H}]^{+}$1515.9961, found 1515.9968.

\section{2 .8 化合物 $\mathbf{B A 2}$ 的合成}

将化合物 $\mathbf{C 1 7}$ (98.1 mg, $0.2 \mathrm{mmol}$ )、化合物 $\mathbf{C 1 8}$ (106.3 mg, $0.2 \mathrm{mmol}$ )、 $1 \mathrm{mg}$ 的 $\mathrm{P}_{2} \mathrm{O}_{5}$ 、对甲基苯磺酸 $(5 \mathrm{mg}$, $0.029 \mathrm{mmol}$ )和 $10 \mathrm{~mL}$ 的甲苯置于 $100 \mathrm{~mL}$ 的三口瓶中. 在 $\mathrm{N}_{2}$ 保护下, 于 $120{ }^{\circ} \mathrm{C}$ 油浴锅下搅拌 $12 \mathrm{~h}$. 冷却至室 温, 采用二氯甲烷和饱和食盐水萃取, 无水硫酸镁干燥, 过滤, 旋干溶剂得粗产品. 进一步, 采用硅胶色谱柱提 纯 [洗脱剂为 $V$ (石油醚)： $V$ (二氯甲烷 $)=1 ： 1$ ] 得 130.6 $\mathrm{mg}$ 紫色固体 BA2, 产率 43\%. m.p. $>300{ }^{\circ} \mathrm{C} ;{ }^{1} \mathrm{H} \mathrm{NMR}$ $\left(400 \mathrm{MHz}, \mathrm{CDCl}_{3}\right) \delta: 9.55(\mathrm{~d}, J=8.0 \mathrm{~Hz}, 2 \mathrm{H}), 9.20(\mathrm{~d}, J=$ $8.0 \mathrm{~Hz}, 2 \mathrm{H}), 8.49$ (d, $J=8.0 \mathrm{~Hz}, 2 \mathrm{H}), 8.35$ (d, $J=8.0 \mathrm{~Hz}$, $2 \mathrm{H}), 8.01$ (d, $J=8.0 \mathrm{~Hz}, 2 \mathrm{H}), 7.94$ (d, $J=8.0 \mathrm{~Hz}, 2 \mathrm{H}), 3.95$ (br, 8H), 2.10 (br, 2H), 1.88 (br, 2H), $1.50 \sim 12.1(\mathrm{~m}, 80 \mathrm{H})$, $1.08(\mathrm{t}, J=8.0 \mathrm{~Hz}, 6 \mathrm{H}), 0.98(\mathrm{t}, J=8.0 \mathrm{~Hz}, 6 \mathrm{H}), 0.88 \sim$ $0.83(\mathrm{~m}, 12 \mathrm{H}) ;{ }^{13} \mathrm{C} \mathrm{NMR}\left(100 \mathrm{MHz}, \mathrm{CDCl}_{3}\right) \delta: 192.1$, $167.9,163.0,162.8,157.9,154.9,139.1,138.1,137.7$, $137.0,134.3,132.6,131.7,131.0,129.6,125.7,124.9$, $122.4,122.0,116.7,115.8,44.6,43.6,37.9,36.7,32.0$, $31.9,31.7,30.9,30.1,29.7,29.7,29.6,29.4,28.8,26.5$, 24.3, 23.2, 22.7, 14.3, 14.1, 10.9. HRMS (MALDI) calcd for $\mathrm{C}_{98} \mathrm{H}_{129} \mathrm{~N}_{6} \mathrm{O}_{8}[\mathrm{M}+\mathrm{H}]^{+}$: 1517.9866 , found 1517.9877 . 
辅助材料(Supporting Information) A2 的晶体基本参 数, BA1 和 BA2 的 TGA 和 DSC 曲线, 吸收光谱对比图, 异靛蓝分子的 CV 曲线, BA1 和 BA2 薄膜 OFET 器件的 输出曲线以及所得新化合物的 ${ }^{1} \mathrm{H} \mathrm{NMR} /{ }^{13} \mathrm{C} \mathrm{NMR}$ 谱图. 这些材料可以免费从本刊网站(http://sioc-journal.cn/)上 下载.

\section{References}

[1] Bharitkar, Y. P.; Datta, S.; Sett, S.; Marathee, N.; Khan, P.; Mondal, N. B. Chem. Biol. Interact. 2017, 7, 19.

[2] Mei, J.; Graham, K. R.; Stalder, R.; Reynolds, J. R. Org. Lett. 2010, 12,660 .

[3] Papageorgiou, C.; Borer, X. Helv. Chim. Acta 1988, 71, 1079

[4] Ding, L.; Wang, Z. Y.; Wang, J. Y.; Pei, J. Chin. J. Chem. 2020, 38, 13.

[5] Lu, Y.; Ding, Y. F.; Wang, J. Y.; Pei, J. Chin. J. Org. Chem. 2016, 36, 2272 (in Chinese) (卢阳，丁一凡，王婕妤，裴坚，有机化学, 2016, 36, 2272.)

[6] (a) Wang, E. G.; Mammo, W.; Andersson, M. R. Adv. Mater. 2014, 26,1801 .

(b) Chen, H. J. Chin. J. Org. Chem. 2016, 36, 460 (in Chinese) (陈华杰, 有机化学, 2016, 36, 2272.)

[7] Estrada, L. A.; Liu, D. Y.; Salazar, D. H.; Dyer, A. L.; Reynolds, J. R. Macromolecules 2012, 45, 8211.

[8] Bogdanov, A.; Mironov, V.; Musin, L.; Musin, R. Synthesis 2010, 3268.

[9] El-Kateb, A.; Hennawy, I.; Shabana, R.; Osman, F. Phosphorus, Sulfur Relat. Elem. 1984, 20, 329.

[10] Zhang, G. B.; Fu, Y. Y.; Xie, Z. Y.; Zhang, Q. Macromolecules 2011, 44, 1414

[11] Cao, Y. Y.; Dou, J. H.; Zhao, N. J.; Zhang, S. M.; Zheng, Y. Q.; Zhang, J. P.; Wang, J. Y.; Pei, J.; Wang, Y. P. Chem. Mater. 2017, 29, 718.

[12] Ashraf, R. S.; Kronemeijer, A. J.; James, D. I.; Sirringhaus, H.; McCulloch, I. Chem. Commun. 2012, 48, 3939.

[13] Li, C. C.; Un, H. I.; Pei, J. W.; Cai, M.; Wang, X.; Wang, J. Y.; Lan, Z. G.; Pei, J.; Wan, X. B. Chem. Eur. J. 2018, 24, 9807.

[14] Huang, J. Y.; Mao, Z. P.; Chen, Z. H.; Gao, D.; Wei, C. Y.; Zhang, W. F.; Yu, G. Chem. Mater. 2016, 28, 2209.

[15] Gao, Y.; Deng, Y. F.; Tian, H. K.; Zhang, J. D.; Yan, D. H.; Geng, Y. H.; Wang, F. S. Adv. Mater. 2017, 29, 1606217.

[16] Yang, J.; Zhao, Z. Y.; Geng, H.; Cheng, C. L.; Chen, J. Y.; Sun, Y. L.; Shi, L. X.; Yi, Y. P.; Shuai, Z. G.; Guo, Y. L.; Wang, S.; Liu, Y. Q. Adv. Mater. 2017, 29, 1702115.

[17] Lei, T.; Dou, J. H.; Ma, Z. J.; Liu, C. J.; Wang, J. Y.; Pei, J. Chem. Sci. 2013, 4, 2447.

[18] Hasegawa, T.; Ashizawa, M.; Matsumoto, H. RSC Adv. 2015, 5, 61035.

[19] Xu, S. L.; Ai, N.; Zhao, N.; Lan, Z. G.; Wang, X.; Pei, J.; Wan, X. B. RSC Adv. 2015, 5, 8340.

[20] Wan, Y.; Ashraf, R. S.; Nielsen, C. B.; Collado-Fregoso, E.; Niazi, M. R.; Yousaf, S. A.; Kirkus, M.; Chen, H. Y.; Amassian, A.; Durrant, J. R.; McCullon, I. Adv. Mater. 2015, 27, 4702.

[21] Jiang, Y.; Zheng, X. H.; Deng, Y. F.; Tian, H. K.; Ding, J. Q.; Xie, Z. G.; Geng, Y. H.; Wang, F. S. Angew. Chem., Int. Ed. 2018, 57, 10283.

[22] Lei, T.; Dou, J. H.; Cao, X. Y.; Wang, J. Y.; Pei, J. J. Am. Chem. Soc. 2013, 135, 12168 .
[23] Ma, S. X.; Zhang, G. B.; Wang, F. F.; Dai, Y. R.; Lu, H. B.; Qiu, L. Z.; Ding, Y. S.; Cho, K. Macromolecules 2018, 51, 5704.

[24] He, Y.; Quinn, J.; Deng, Y.; Li, Y. Org. Electron. 2016, 35, 41

[25] Jiang, Y.; Gao, Y.; Tian, H. K.; Ding, J. Q.; Yan, D. H; Geng, Y. H.; Wang, F. S. Macromolecules 2016, 49, 2135.

[26] Mei, J. G.; Kim, D. H.; Ayzner, A. L.; Toney, M. F.; Bao, Z. N. J. Am. Chem. Soc. 2011, 133, 20130.

[27] Lei, T.; Dou, J. H.; Pei, J. Adv. Mater. 2012, 24, 6457

[28] Mei, J. G.; Wu, H. C.; Diao, Y.; Appleton, A.; Wang, H.; Zhou, Y.; Lee, W. Y.; Kurosawa, T.; Chen, W. C.; Bao, Z. N. Adv. Funct. Mater. 2015, 25, 3455.

[29] Lei, T.; Wang, J. Y.; Pei, J. Acc. Chem. Res. 2014, 47, 1117.

[30] Lan, L. Y.; Chen, Z. M.; Ying, L.; Huang, F.; Cao, Y. Org. Electron. 2016, 30, 176.

[31] Li, X. L.; Guo, J.; Yang, L. F.; Chao, M. H.; Zheng, L. P; Ma, Z. Y.; Hu, Y. Y.; Zhao, Y.; Chen, H. J.; Liu, Y. Q. Front. Chem. 2019, 7, 362.

[32] Li, H. Y.; Kim, F. S.; Ren, G. Q.; Hollenbeck, E. C.; Subramaniyan, S.; Jenekhe, S. A. Angew. Chem., Int. Ed. 2013, 52, 5513.

[33] Li, H. Y.; Kim, S.; Ren, G. Q.; Jenekhe, S. A. J. Am. Chem. Soc. 2013, 135, 14920.

[34] Li, H. Y.; Earmme, T.; Ren, G. Q.; Saeki, A.; Yoshikawa, S.; Murari, N. M.; Subramaniyan, S.; Crane, M. J.; Seki, S.; Jenekhe, S. A. J. Am. Chem. Soc. 2014, 136, 14589.

[35] Hwang, Y. J.; Li, H. Y.; Courtright, B. A. E.; Subramaniyan, S.; Jenekhe, S. A. Adv. Mater. 2016, 28, 124.

[36] Kolhe, N. B.; West, S. M.; Tran, D. K.; Ding, X. M.; Kuzuhara, D.; Yoshimot, N.; Koganezawa, T.; Jenekhe, S. A. Chem. Mater. 2020 , 32, 195

[37] Qian, X.; Zhu, Y. Z.; Song, J.; Gao, X. P.; Zheng, J. Y. Org. Lett. 2013, 15, 6034.

[38] Zhao, D.; Hu, J. Y.; Liu, Z. J.; Xiao, B.; Wang, X. Z.; Zhou, E. J.; Zhang, Q. Dyes Pigm. 2018, 151, 102.

[39] Jiro, T.; Tong, Z. W.; Yasuji, I. ARKIVOC 2001, 67.

[40] Echegaray, P. D.; Mancheño, M. J.; Arrechea-Marcos, I.; Juárez, R.; López-Espejo, G.; Navarrete, J. T. L.; Ramos, M. M.; Seoane, C.; Ortiz, R. P.; Segura, J. L. J. Org. Chem. 2016, 81, 11256.

[41] Ding, L.; Yang, C. Y.; Su, Z. M.; Pei, J. Sci. China Chem. 2015, 58, 364.

[42] Chen, H. J.; Cai, G. S.; Guo, A. K.; Zhao, Z. Y.; Kuang, J. H.; Zheng, L. P.; Zhao, L. L.; Chen, J. Y.; Guo, Y. L.; Liu, Y. Q. Macromolecules 2019, 52, 6149 .

[43] Frisch, M. J.; Trucks, G. W.; Schlegel, H. B.; Scuseria, G. E.; Robb, M. A.; Cheeseman, J. R.; Scalmani, G.; Barone, V.; Mennucci, B.; Petersson, G. A.; Nakatsuji, H.; Caricato, M.; Li, X.; Hratchian, H. P.; Izmaylov, A. F.; Bloino, J.; Zheng, G.; Sonnenberg, J. L.; Hada, M.; Ehara, M.; Toyota, K.; Fukuda, R.; Hasegawa, J.; Ishida, M.; Nakajima, T.; Honda, Y.; Kitao, O.; Nakai, H. Vreven, T.; Montgomery, J. A.; Jr., Peralta, J. E.; Ogliaro, F.; Bearpark, M.; Heyd, J. J.; Brothers, E.; Kudin, K. N.; Staroverov, V. N.; Kobayashi, R.; Normand, J.; Raghavachari, K.; Rendell, A.; Burant, J. C.; Iyengar, S. S.; Tomasi, J.; Cossi, M.; Rega, N.; Millam, J. M.; Klene, M.; Knox, J. E.; Cross, J. B.; Bakken, V.; Adamo, C.; Jaramillo, J.; Gomperts, R.; Stratmann, R. E.; Yazyev, O.; Austin, A. J.; Cammi, R.; Pomelli, C.; Ochterski, J. W.; Martin, R. L.; Morokuma, K.; Zakrzewski, V. G.; Voth, G. A.; Salvador, P.; Dannenberg, J. J.; Dapprich, S.; Daniels, A. D.; Farkas, Ö.; Foresman, J. B.; Ortiz, J. V.; Cioslowski, J.; Fox, D. J. Gaussian 09, Revision A.02, Gaussian, Inc, Wallingford CT, 2009.

[44] Cao, Q. F.; Xiong, W. T.; Chen, H. J.; Cai, G. S.; Wang, G.; Zheng, L. P.; Sun, Y. M. J. Mater. Chem. A 2017, 5, 7451. 